\title{
An Overview of Bioactive Natural Products-Based Nano-Drug Delivery Systems in Antitumor Chemotherapy
}

\author{
Yihao $\mathrm{Niu}^{1}$ \\ ${ }^{1}$ Hebei University of Chinese Medicine, 050200
}

\begin{abstract}
Many bioactive natural products are derived from traditional Chinese medicine. Some of them showed good treatment potential in tumor chemotherapy. However, limited by the poorly solubility and non-selective toxicity of most of bioactive natural products, their antitumor efficacy could not be further applied in clinicals. Nano-drug delivery systems provided good platforms for bioactive natural products in antitumor treatment, and many tumor microenvironment-responsive nano-delivery carriers facilitated them to achieve more accurate tumor targeting and less toxicity, and finally improved antitumor ability of bioactive natural products.
\end{abstract}

\section{Introduction}

Cancer is one of the most essential health-related issues globally. In traditional Chinese Medicines, Essential Medical Manual said: "Accumulating so much forms lump merely because healthy Qi is insufficient, and then evil Qi are occupying the body."

Traditional Chinese medicine held the view that tumor was a visual lump caused by that healthy Qi was insufficient, dysfunction of viscera, Stagnation of Qi movement, and phlegm-fluid generates toxicity and then stays longer in the body. Clinically, surgery, radiotherapy, chemotherapy, immunotherapy and Traditional Chinese Medicine were often used to treat tumor proliferation.

Chemotherapy, as one of the conventional antitumor treatment, was a method that utilize chemo-drugs to prevent the proliferation, infiltration, and metastasis of cancer cells, and ultimately kill cancer cells. It was appropriate for systemic treatment, and has a therapeutic effect on primary and metastatic lesions. Many researchers were dedicated to discover bioactive natural products in antitumor chemotherapy, because nature has always been the most important source of bioactive antitumor drugs1. Commonly known bioactive natural antitumor drugs included paclitaxel, camptothecin, vincristine, EGCG, curcumin, Doxorubicin and so on2, 3. Different bioactive natural products had different mechanisms of action. They directly kill cancer cells through distinct pathways and targets, including tumor cells, tumor tissues, extracellular matrix, immune system, blood vessels. They showed good antitumor effects at the cellular level. Some antitumor drugs derived from natural products have been successfully developed and marketed and used clinically3. However, most natural antitumor drugs were poorly water-soluble, and low bioavailability and short half-life, which limited their further application. Nano-drug delivery systems based on bioactive natural drugs have been reported to increase the solubility of drugs and improve tumor targeting, thereby improving the therapeutic effects of bioactive natural antitumor drugs and reducing systemic toxicity. In addition, based on the complicated tumor microenvironment including abnormal $\mathrm{pH}$, high intracellularly reducibility, and highly-expressing enzymes, researchers have further designed bioactive natural drug nano-delivery system that in response to the tumor microenvironment. This review summarized some antitumor drugs derived from bioactive natural medicines and illustrated their mechanisms of action, as well as some antitumor nanomedicines derived from natural medicines which have been used in clinical practice. In addition, tumor microenvironment responsive antitumor nano-delivery systems of bioactive natural products were also briefly described.

\section{Bioactive natural products in antitumor chemo-therapy}

Natural products mainly included polysaccharides, amino acids, antibiotics, alkaloids, flavonoids, terpenoids and other chemical components. Antitumor drugs derived from plants have become an important part of clinical antitumor drugs. At present, many bioactive natural products such as vinblastine, vincristine, paclitaxel, docetaxel, irinotecan, hydroxylcamptothecin have been applied clinically. Moreover, curcumin, quercetin, EGCG and other active ingredients derived from natural products have been in clinical trials for antitumor therapies.

Many antitumor natural products could perform direct killing functions in tumor cells, and they have distinct mechanisms.

\footnotetext{
* Corresponding author: nyh18632138657@163.com
} 
Camptothecin is an important natural alkaloid, which is isolated from the root bark of the unique Davidia family plant Camptotheca acuminate, and it showed significant antitumor activity. It could bind to the covalent compounds formed by topo I and DNA to form ternary complexes that inhibit DNA replication and ultimately lead to cell death ${ }^{4}$. Their derivates such as 10 hydroxycamptothecin, topotecan, irinotecan and belotecan have been commonly used clinically to treat colon cancer, small-cell lung cancer and ovarian cancer. However, further research found that camptothecin had strong side effects, such as bone marrow suppression and bladder toxicity. In addition, the disadvantage of poor water solubility and poor stability also severely restricted the clinical anti-tumor treatment of camptothecin and its derivates.

Paclitaxel was first extracted from the bark of Taxus brevifolia. Paclitaxel promoted tubulin polymerization and assembly, and prevents their depolymerization, thereby stabilizing microtubules and inhibiting the mitosis of cancer cells and preventing the induction of apoptosis, thereby effectively preventing the proliferation of cancer cells and exerting anti-tumor effects $^{5}$. It showed excellent antitumor ability in many cancers. However, like other bioactive natural products, very poor solubility limited their further application ${ }^{5}$.

Artemisinin and its derivates, known as an antimalaria drug, also show good antitumor potential. Tumor cells had a high metabolism of nucleic acid and abundant expression of the transferrin receptor on the surface. After binding to the receptor, transferrin increased intracellular iron content through receptor-mediated uptake. Therefore, the iron content of tumor cells was higher than that of normal cells. The peroxide bridge structure of artemisinin broke after reacting with ferrous ions, generating carbon-based free radicals or reactive oxygen species (ROS). These free radicals could play an antitumor role by promoting apoptosis, inhibiting cell proliferation, angiogenesis and direct DNA damage. In addition, artemisinin and its derivates could induce ferroptosis of tumor cells and inhibit angiogenesis ${ }^{6}$.

Vincristine mainly acted on tubulin of tumor cells and interfering with tumor cell metabolism. Binding to tubulin, which stopped metaphase cell division and lead to an increase in mitotic cell populations and stop cell division. It could also interfere with protein metabolism and inhibit the activity of RNA polymerase, and change the lipid composition of the tumor cell membrane. Vincristine mainly used for the treatment of acute lymphoblastic leukemia, breast cancer, malignant lymphoma and small cell lung cancer and other diseases ${ }^{7}$.

In summary, although these bioactive natural products showed good antitumor efficacy in different mechanisms, their limitations were still existed such as strong toxicity, short half-time and poor solubility, which could limit their further clinical application.

\section{Bioactive natural products-based nano-drug delivery systems in antitumor chemotherapy}

\section{1 brief introduction of nano-drug delivery systems}

In recent years, nano-drug delivery systems showed great potential in improving the antitumor efficacy of bioactive natural products.

Nano-drug delivery systems usually are within a particle size range of $100 \mathrm{~nm}-200 \mathrm{~nm}$. Due to their small size and large surface area, they have a huge surface energy. Compared with free materials, there were key differences and biophysical properties related to their particle size. Small-molecule drugs, large-molecule proteins, and genetic drugs could be transported by adsorbing, binding, dispersing or encapsulating the drugs with the aid of carrier materials. Nanocarriers could increase the half-life of drugs by protecting them from degradation, and improve the solubility and delivery efficiency of poorly soluble drugs.

Tumors have complicated microenvironments. Due to the rapid proliferation of tumor cells, there were large pores between the vascular epithelial cells at the tumor site, lacking the ordered branching hierarchy from large vessels to continuous smaller vessels. Vascular space distribution of tumor was uneven, dilated and tortuous, leaving various sizes of non-vascular space and with irregular blood flow velocity. At the same time, the tumor tissue lymphatic vessels were incomplete and lymphatic circulation was strongly limited. Owing to the specific environment, nano-drugs with proper size and characteristics could selectively permeate and accumulate there and could hardly leak from lymphatic systems. Therefore, nano-drugs could achieve targeting functions. This phenomenon called EPR effect (enhanced permeability and retention effect) was reported to happen in tumor sites ${ }^{8}$. This was also the theoretical basis for the passive targeting of nanomedicine, and this was called passive targeting.

Aside from passive targeting, compared with normal cells, there were many highly expressed receptors such as folate receptors on the surface of tumor cells, therefore, the modification of specific targeted molecular ligands on the surface of nano-drugs could improve the targeting ability. This was called active targeting.

There were many kinds of nano-drug delivery systems, such as liposomes, polymeric nanoparticles, micelles, nanocrystals, metal-based nanocarriers and other inorganic nanoparticles such as carbon tubes. Some of them have been in clinicals.

\subsection{Advantages of bioactive natural nano-drug delivery systems in antitumor therapy}

Nano-drugs could solve various obstacles in bioactive natural drug delivery. First of all, the water solubility of insoluble drugs could be increased with nano-structures. In addition, drugs could target to specific cells or organs to increase efficacy, meanwhile reducing toxicity of 
normal tissues. Moreover, nanocarriers could change the pharmacokinetics of free drugs and prolong their releasing time. Also, many vulnerable and hydrophilic drugs such as nucleic acid, peptides and proteins could be protected by nanocarriers and improve their uptake into certain cells. In some cases, nanocarriers could perform as an immune-regulating regent.

\subsection{Nano-drugs in clinical use}

\subsubsection{Liposomes}

Liposomes possessed many properties such as high drug loading efficiency, good biocompatibility, and good stability, and they were commonly used in clinicals nowadays. Doxorubicin was a kind of anthracycline antibiotic drug, which has a good effect on cancer treatment. It could induce insertion of DNA and destruction of topoisomerase II, mediating DNA repair and free radical generation, causing damage to cell membranes, DNA and proteins of tumor cells. However, it was noted that doxorubicin could cause serious and potentially life-threatening heart problems. Therefore, Doxil ${ }^{\circledR}$ (PEGylated doxorubicin liposomes), the first FDA-approved nano-drug(1995), was adopted to treat cancer including metastatic ovarian cancer and AIDrelated Kaposi's sarcoma. It balanced the efficacy and safety of doxorubicin, while ensuring efficacy and reducing toxicity. With liposome encapsulating and PEGylation, the circulation time in the body could be prolonged and drug targeting efficiency could also increase ${ }^{9}$.

Natural bioactive products-based liposomes have also been applied in clinicals. Vincristine sulfate could bind to tubulin and changed the balance of tubulin polymerization, resulting in changes in the structure and function of tubulin. Vincristine sulfate stabilized the structure and prevented chromosome separation, causing metaphase stagnation and inhibiting mitosis, finally inhibiting tumor cell proliferation. Vincristine sulfate liposome (Marqibo ${ }^{\circledR}$ ) could reduce its side-effect on normal tissues and improve safety ${ }^{10}$.

Another example was irinotecan. Irinotecan was a semi-synthetic water-soluble camptothecin derivative with short half-life, poor stability and high toxicity. By liposomes encapsulating, the lactone ring in the structure of irinotecan could be protected from hydrolysis. At the same time, the circulation time and half-life in vivo were enhanced by nano-encapsulating. Irinotecan liposome (Onivyde ${ }^{\circledR}$ ) was approved for marketing by FDA in October 2015 for the treatment of advanced pancreatic cancer.

\subsubsection{Polymeric Micelles}

Polymeric micelles have a longer circulation time in the body and reduced immunogenicity, which could protect drug stability and possess longer half-life, and they could reduce toxicity by encapsulating or physically embedding a drug in a polymer matrix, decreasing its interaction with healthy cells.
Taxol was the first marketed paclitaxel preparation. The solubility was improved by using Cremophore EL ${ }^{\circledR}$ and ethanol as solubilizer. While this preparation showed severe dose-related side-effect due to the allergic reaction caused by Cremophore EL ${ }^{\circledR}$. Paclitaxel-based nano-preparation was aim to reduce these side-effects.

Genexol-PM ${ }^{\circledR}$ (Paclitaxel micelles) was an injective micellar paclitaxel developed by Samyang, South Korea in 2007. it was formed by paclitaxel and Polyethylene glycol - polyethylene lactide(PEG-PLA) and did not contain Cremophore EL ${ }^{\circledR}$. PEG, s the coat of polymer micelles, could avoid the phagocytosis of the reticuloendothelial system and prolong the circulation time in vivo, and the preparation showed a certain passive targeting effect and reduced allergic reactions ${ }^{[11-}$ 12].

\subsubsection{Albumin-based nano-preparations}

Albumin-bounded paclitaxel (Abraxane $\AA$, nab-paclitaxel, Celgene, USA) were widely applied in clinicals nowadays. Albumin was a kind of ideal nano-drug carrier owing to its non-toxicity and lowimmunogenicity. Abraxane was a nano-combination of albumin and insoluble paclitaxel with size of around 130 $\mathrm{nm}$. Because of lacking Cremophor EL ${ }^{\circledR}$, the tolerated dose was enhanced and allergic reactions were reduced compared with conventional injections. In addition, no premedication or special syringe was required for the administration of the drug ${ }^{13}$. It was approved by the FDA in 2005 for the treatment of breast cancer, non-small cell lung cancer and pancreatic cancer. However, the price of Abraxane was expensive, the requirements of the site environment and personnel quality were relatively high.

\section{Strategies in tumor microenvironment responsive nano-drug delivery systems loading with bioactive natural products}

\subsection{In vivo bio-fate of nano-drug delivery systems targeting to tumors}

Regardless of the fact that many nano-drugs derived from natural products were in clinical applications, there were still many obstacles existed in nano-drug delivery systems in antitumor therapies due to the complicated tumor microenvironment.

In addition to targeting tumor cells, nano-drugs could also target tumor vascular endothelial cells and immune cells in the tumor microenvironment. In order to achieve a satisfactory therapeutic effect, nano-drugs also need to pass through intracellular transport, and in response to a certain environment in the cell, the structure was dissolved, thereby releasing the drug molecules, and finally playing an anti-tumor role.

First of all, in blood circulation, positively electric or hydrophobic nanoparticles were easy to absorb plasma proteins. A large number of immune cells in the blood, such as macrophages and neutrophils, could recognize proteins on the surface of nano-drugs and activate the 
non-specific phagocytic function of related immune cells to finally eliminate them from the blood. Secondly, the particle size of nanomedicine was also related to its cycling path. When the nanodrug was less than $5 \mathrm{~nm}$, it could penetrate through the vascular wall through extravasation, and the glomerular capillary wall of the kidney participates in the purification of the compound from systemic circulation, which was quickly cleared by the kidney for preparation in vitro. When the nanodrug was larger than $15 \mathrm{~nm}$, it could be removed by capillary mechanical filtration. At the same time, nano-drugs with unsuitable particle sizes would over-accumulate in the liver, spleen, lymph nodes and lungs, and cause toxicity to organs and tissues. It was reported that particles with diameters $<200 \mathrm{~nm}$ are more effective for accumulating in tumor tissues14. Therefore, surface hydrophilic and negatively or electrically neutral particles could achieve a longer period of blood circulation, increasing the chance and probability of targeting tumor tissues.

Due to poor lymphoid circulation inside the tumor and low drainage efficiency, tumor tissues have high interstitial pressure, which makes it difficult for external drugs and nanoparticles to diffuse into the tumor tissues, while cells and related cytokines in the tumor tissues were easy to diffuse out, leading to metastasis of the tumor.

Meanwhile, in the pathological conditions of tumors, the extracellular matrix (ECM) was dense, which promoted the invasion and circulation of tumor cells from the primary site and the recruitment of endothelial cells to promote tumor angiogenesis and tumor growth, but also restricted further spread and penetration of nanomedicine after it reached the tumor site, thus unable to play a good antitumor efficacy.

Even when nano-drugs finally reached tumor cells, endothelial tight connection and intracellular barriers also limited their entry. The cell membrane was a lipid bilayer, so the surface hydrophilic or negative or neutral nanocarriers were not easy to be ingested by tumor cells. Even through endocytosis into tumor cells, due to lysosomal degradation, and so on, so that the efficacy was not good.

\subsection{Tumor physiological microenvironment}

In tumor tissues, special physiological microenvironment included abnormal $\mathrm{pH}$ value in tumors, intracellular reductive environment, highly-expressing enzymes in tumor tissue, and intracellular highly reactive oxygen species (ROS). These special characteristics of tumor tissues and tumor cells could be utilized to design rational stimulus-responsive antitumor nano-delivery systems for bioactive natural products.

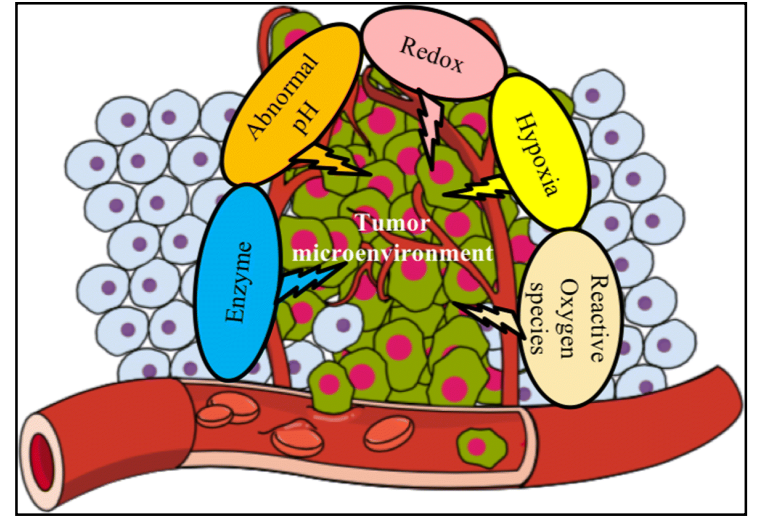

Figure 1. Characteristics of tumor microenvironment ${ }^{15}$

\subsection{1 $\mathrm{pH}$}

Compared with $\mathrm{pH} 7.4$ in normal tissues and blood circulation, it was reported that the $\mathrm{pH}$ in tumor tissue was around 6.5-7.2, in addition, the intracellular $\mathrm{pH}$ in tumor cells was lower. It was found that the $\mathrm{pH}$ in early endosomes was around 6.0 and in lysosomes was only 4.0-5.0. The mildly-acidic microenvironment was mainly due to lactic acid production in tumor cells because of the rapid proliferation and increased aerobic and anaerobic glycolysis of tumor cells 16 .

Because of the $\mathrm{pH}$ difference in normal tissues and tumors, researchers have designed many $\mathrm{pH}$-responsive anti-tumor nano-drug delivery systems for natural bioactive products to achieve more accurate targeting and drug releasing in tumor cells. The $\mathrm{pH}$ change could trigger drug release of nanoparticles intracellularly.

When the $\mathrm{pH}$ reached a certain critical value such as 6.5 , the type and the charge of the material molecules may change, and the electrostatic force between the material molecules also changed, therefore, $\mathrm{pH}$-sensitive nanocarrier could expand or dissolve, thereby releasing the loading anti-tumor drugs.

Hydroxycamptothecin (HCPT) was a kind of bioactive natural indole alkaloid which was isolated from Camptotheca acuminata Decne. It showed potent anticancer effects and low toxicity while the solubility was relatively poor and it was structurally-unstable under physiological conditions. Therefore, researchers developed a pH-sensitive polymeric nano-micellar system to increase their solubility and tumor targeting efficacy ${ }^{17}$. In this research, $\mathrm{pH}$-sensitive material monomethyl ether-poly(ethylene glycol)-iminopoly(benzyl-L-aspartate) (PIPA) was utilized to form $\mathrm{pH}$-sensitive micelles encapsulating poorly-soluble HCPT. The average diameter of HCPT-loaded PIPA micelles was around $230 \mathrm{~nm}$, exhibiting good colloid stability, storage stability and plasma stability. In in vitro drug releasing experiment, PIPA micelles showed good $\mathrm{pH}$ sensitivity. compared with $\mathrm{pH}$ 7.4, PIPA micelles showed relatively faster drug releasing profile in $\mathrm{pH} 6.0$ and $\mathrm{pH} 5.0$, achieving $65 \%$ and $75 \%$ releasing of HCPT within $24 \mathrm{~h}$. as a comparison, non-pH sensitive PPA micelles showed modest drug releasing profile of 30\% with $24 \mathrm{~h}$. In addition, compared with commercial free HCPT, PIPA micelles exhibited improved cytotoxicity in 
tumor cell lines including HepG2 and MCF-7 cells. In vivo, the intravenous administration of PIPA micelles showed higher plasma concentration than that of free HCPT solution. Moreover, in tumor inhibition experiment of $\mathrm{H} 22$ tumor-bearing mice, compared with saline group, the tumor volume and weight were significantly decreased and the tumor inhibition rates of pH-sensitive PIPA micelles was $63.30 \%$, achieving the best antitumor efficacy. Compared with free HCPT, it showed more tumor retention of HCPT and less systemic toxicity in PIPA micelles group, showing good safety.

In another research, a novel $\mathrm{pH}$-sensitive cleavable nano-drug delivery liposome (CL-Lip) encapsulating bioactive natural product paclitaxel was designed ${ }^{18}$. Before CL-Lip administration, free losartan was injected to reduce type I collagen in the extracellular matrix and facilitate antitumor drug permeation. The CL-Lips consisted of $\mathrm{pH}$-sensitive PEG5K-Hydrazone-PE and cell membrane permeable DSPE-PEG2K-R8. When liposomes arrived at tumor sites by EPR effect, and due to the $\mathrm{pH}$-sensitive group of hydrazone, the PEG5K could be releasing and the R8 peptide was exposed to increasing the cell penetration efficacy.

In vitro experiments, the particle size of CL-Lips loaded with PTX was about $120 \mathrm{~nm}$, the particle size was uniform, and the PDI was small. The negative potential reflected the long-cycle effect of PEG modification, and the stability in vitro was good. The in vitro releasing results showed that compared with free drug, CL-lips showed the sustained release effect, and the results showed that the R8 peptide could be exposed within tumor sites because PEG5K could be hydrolyzed in lower $\mathrm{pH}$. In addition, in vitro experiments, this nanodrug delivery system overcame the shortcomings of poor internalization ability of PEG, and improved the targeting ability and internalization ability of the drug delivery system.

In in vivo experiments, compared with the Cl-Lip group, the tumor-bearing mice with the administration of free losartan and the Cl-Lips had the best tumor suppressive effect. The tumor volume became smaller and the tumor weight decreased. At the same time, compared with the high consumption of free paclitaxel in vivo, the low efficacy on tumor cell growth inhibition, its own neurotoxicity and hypersensitivity defects, the combined drug delivery system of losartan and paclitaxel $\mathrm{pH}$-sensitive cleavable liposomes was more effective with less toxicity, higher safety, and better antitumor efficacy in inhibiting tumor cell growth and metastasis.

\subsection{2 enzymes}

Compared with normal cells, tumor cells have problems in the mechanism of controlling enzyme activity, which leads to significant differences in the expression of various enzymes between tumor cells and normal cells. An example was matrix metalloproteinase (MMPs), which were highly-expressed in many kinds of tumor types. They were mainly expressed in ECM, and they could degrade ECM by affecting Collagen IV and laminin, thereby facilitating tumor cells to cross the matrix membrane barrier, infecting surrounding tissues and causing metastasis ${ }^{19}$. MMP also played a major role in tumor proliferation, inflammatory response, and cell apoptosis. MMP2 and MMP9 were found in a variety of tumor cells, including gastric cancer, breast cancer, prostate cancer, rectal cancer, lung cancer and ovarian cancer. Therefore, MMP2 and MMP9 were often selected as targets for targeting of the tumor microenvironment to improve antitumor efficacy ${ }^{20}$.

Researchers designed a tumor-targeting MMP2sensitive polymeric micelle loading paclitaxel as model drug ${ }^{19}$. A PEG2000-paclitaxel conjugate with a MMP2sensitive octapeptide, TATp-PEG1000-PE, and PEG1000-PE were exploited to form the "core-shell" micelles via self-assembling in an aqueous environment. Once administration, micelles could selectively accumulate in tumor sites, and the MMP2-sensitve octapeptide was cleaved by locally high-expressing MMP2, and the cell-penetrating peptide TATp would be exposed to facilitate cell uptake.

Preparation and characterization of micelles showed that the micelles had uniform shape, strong hydrophobic properties and small particle size, which reflected the long cycle effect of PEG modification and prolonged the half-life. The stable and hydrophobic core-shell structure allows it to be stored for a long time in aqueous solution to avoid proteolysis. The linker sensitive to MMP2 encounters MMP2 breakage and releases the drug, thereby increasing the permeability of the nano-system. Compared with free PTX, the nano-system was stable in the blood circulation system, and could release more in tumor cells, which indicated the sustained release effect by nanocarriers.

The toxicity results showed that it was less toxic than free PTX due to PEGlytion. Only part of the released PTX could be effectively absorbed, which directly prolongs the action time of PTX. The results of in vitro experiments showed that compared with free PTX and control groups, this system has a relatively high drug load, a lower risk of drug leakage, and improved pharmacokinetics and efficacy.

In in vivo experiments, compared with the control group of non-sensitive nano-preparations, the experimental group of MMP-sensitive polymeric micelles in the experiment of tumor-bearing mice had better tumor inhibition effect, showing significantly inhibited and reduced tumor volume and weight. Cell uptake mediated by MMP2, moderate size and PEGylation also enable nanoparticles exhibiting better tumor targeting.

\subsubsection{Intracellular reductive condition}

The concentration of glutathione inside and outside the cell varies significantly. Various organelles in the cell, such as cytosol, mitochondria and nucleus, all contain very high concentrations of glutathione (GSH, 2-10 $\mathrm{mmol} / \mathrm{L}$ ), while the concentration of glutathione outside the cell was only $2-10 \mu \mathrm{mol} / \mathrm{L}^{21}$. Due to severe hypoxia in tumor tissues, and some tumors even contain up to 7 times more glutathione (GSH) than normal tissues, it 
presents a reducing environment. The GSH concentration will be higher in multidrug resistant tumor cells ${ }^{22}$.

In addition, the cytoplasm was the major part of the synthesis of reducing substance glutathione. The intracellular glutathione concentration was much higher than the extracellular concentration, and the reduction potential difference between the inside and outside of the cell was 100 to 1000 times. Because of the huge difference in the content of intracellular and extracellular GSH, GSH has been identified as an ideal intracellular stimulating element that triggers the dissociation of drug carriers and the rapid release of drugs, and this could be used as a strategy to restore environmental response ${ }^{23}$.

In nano-delivery systems, disulfide bonds were classic reduction-responsive groups. Under the action of a certain amount of reducing substances (such as glutathione (GSH), dithiothreitol (DTT) or cysteine (Cys), etc.), the disulfide bond was broken and reduced to a sulfhydryl group.

According to the difference in reduction potential between tumor cells and normal cells and the difference between extracellular and intracellular reduction potentials, the reduction-sensitive nano-formulations based on disulfide bonds undergo conformational transformation or structural changes, thereby promoting the incorporation of drug-carrying systems into cells and increasing Intracellular release of the drug.

Researchers designed a multifunctional reductionsensitive star-shaped micelle system with active targeting ability ${ }^{24}$. The nano-micelles(star-PECLss-FA) was self-assembly by the four-arm PCL-PEG copolymer linked by reduction-sensitive disulfide bonds, and folic acid (FA) group was modified on the micelles to enhance the active targeting ability, because of the high expression of folate receptor (FR) on tumor cells.

In in vitro experiments, the particle was uniform and spherical of size of about $100 \mathrm{~nm}$, and the PDI was relatively small. After adding $10 \mathrm{mM} \mathrm{GSH}$, the structure of these micelles was damaged, showing these polymers have reduction-sensitivity. The blank nanocarrier has low toxicity at $100 \mu \mathrm{g} / \mathrm{ml}$, and the cell survival rate was above $80 \%$, indicating good biocompatibility and low toxicity. In vitro release results showed that compared with non-reductive response micelles, star-PECLss micelles and star-PECLss-FA micelles loading DOX have higher solubility in water and release faster in tumor acid and reducing microenvironment. In addition, free doxorubicin entered cells through passive diffusion, and FA-modified reduction-sensitive micelles could enter cells through folate receptor-mediated endocytosis and the uptake was the improved, reduction-sensitive micelles without FA-modification entered the nucleus less and the non-reduction-sensitive micelles entered the cell less, even unable to enter the cell.

In in vivo experiments, compared with the free DOX and star-PECL micelles control group, the starPECLss-FA micelles experimental group has the best tumor suppression effect in the tumor-bearing mice.

\section{CONCLUSION AND PERSPECTIVE}

In recent years, nanotechnologies have helped numerous bioactive natural products achieve better antitumor efficacy and less toxicity. In addition, many smart nanodelivery systems were designed to response to the specific tumor microenvironment in basic scientific research field, which showed good potential for future antitumor treatment. However, many things need to be further explored, such as the long-term toxicity of the nanocarrier themselves, the in vivo efficiency of EPR effect of nano-drugs. In summary, much more effort should be taken on these fields continuously for better application of our traditional medicines.

\section{Reference}

1.D J.Newman and G M.Cragg . Natural Products As Sources of New Drugs over the 30 Years from 1981 to 2010. Journal of natural products (2012) : 75: 311.

2.D J.Newman and G M.Cragg . Natural Products as Sources of New Drugs from 1981 to 2014. Journal of Natural Products ( 2016) : 629.

3.G MCragg, Grothaus P G and Newman D J. Impact of natural products on developing new anti-cancer agents. J Chemical Reviews (2009) : 109: $3012-$ 43.

4.W J.Slichenmyer, E K.Rowinsky, R C.Donehower and S H.Kaufmann. The Current Status of Camptothecin Analogues as Antitumor Agents. Journal of the National Cancer Institute: 271.

5. S.Malik, R M.Cusidó, M H.Mirjalili , E.Moyano , $\mathrm{J}$.Palazonand M.Bonfill. Production of the anticancer drug taxol in Taxus baccata suspension cultures: A review. J Process Biochemistry (2011) : 46: p.23-34.

6. a E O, a M E M S, a O K, a S S, a M C, a K E, et al. Artemisinin derivatives induce iron-dependent cell death (ferroptosis) in tumor cells. Phytomedicine International Journal of Phytotherapy Phytopharmacology (2015) : 22: 1045-54.

7. Mukherjee, Ashis K, Basu, Sourav, Sarkar, Nabanita, et al. Advances in Cancer Therapy with Plant Based Natural Products. Current Medicinal Chemistry (2001).

8. H.Maeda. The enhanced permeability and retention (EPR) effect in tumor vasculature : the key role of tumor-selective macromolecular drug targeting. Advances in Enzyme Regulation (2001) : 41: 189-207.

9. Y C.Barenholz . Doxil — The first FDA-approved nano-drug: Lessons learned. Journal of Controlled Release (2012) : 160: 117-34.

10. Jeffrey, Silverman, Steven and Deitcher. Marqibo? (vincristine sulfate liposome injection) improves the pharmacokinetics and pharmacodynamics of 
vincristine. Cancer Chemotherapy \& Pharmacology (2013).

11. T Y.Kim, D W.Kim, J Y.Chung, G S.Sang and Bang Y J. Kim TY, Kim DW, Chung JY, Shin SG, Kim SC, Heo DS, Kim NK, Bang YJPhase I and pharmacokinetic study of Genexol-PM, a cremophor-free, polymeric micelle-formulated paclitaxel, in patients with advanced malignancies. Clin Cancer Res 10: 3708-3716. Clinical Cancer Research An Official Journal of the American Association for Cancer Research (2004) : 10: 3708-16.

12. K S.Lee , H C.Chung, S A.Im , Y H.Park, C S.Kim, S B.Kim, et al. Multicenter phase II trial of Genexol-PM, a Cremophor-free, polymeric micelle formulation of paclitaxel, in patients with metastatic breast cancer. Breast Cancer Research \& Treatment (2008) : 108: 241-50.

13. M R.Green , GM.Manikhas, S.Orlov, B.Afanasyev, AM.Makhson, Bhar P, et al. Abraxane((R)), a novel Cremophor((R))-free, albumin-bound particle form of paclitaxel for the treatment of advanced nonsmall-cell lung cancer. Annals of Oncology (2006) : 17: 1263-68.

14. Torchilin and P.Vladimir. Recent advances with liposomes as pharmaceutical carriers. Nature Reviews Drug Discovery ( 2005) .

15. Saji, Uthaman, Kang, Moo, Huh, In-Kyu, et al. Tumor microenvironment-responsive nanoparticles for cancer theragnostic applications. Biomaterials research 2018.

16. E S.Lee, Z.Gao and H B.You . Recent progress in tumor $\mathrm{pH}$ targeting nanotechnology. Journal of Controlled Release (2008) : 132: 164-70.

17. Xiaohui, Pu, Luling, Zhao, Jia, Li, et al. A polymeric micelle with an endosomal $\mathrm{pH}$-sensitivity for intracellular delivery and enhanced antitumor efficacy of hydroxycamptothecin. Acta biomaterialia (2019).

18. High Tumor Penetration of Paclitaxel Loaded $\mathrm{pH}$ Sensitive Cleavable Liposomes by Depletion of Tumor Collagen I in Breast Cancer. Acs Applied Materials \& Interfaces (2015) : 7: 9691.

19. L.Zhu, T.Wang, F.Perche, A.Taigind and V P.Torchilin . Enhanced anticancer activity of nanopreparation containing an MMP2-sensitive PEG-drug conjugate and cell-penetrating moiety. PNAS (2013) : 110: 17047-52.

20. Cathcart J, Pulkoski-Gross A and Cao J. Targeting Matrix Metalloproteinases in Cancer: Bringing New Life to Old Ideas. Genes Dis (2015) : 2: 26-34.

21. R.Cheng, F.Feng, F.Meng, C.Deng, J. Feijen and Z.Zhong . Glutathione-responsive nano-vehicles as a promising platform for targeted intracellular drug and gene delivery. Journal of Controlled Release (2011) : 152: 2-12.
22. A A R, B U H, C T R, D M B and E H M N I. "Smart" materials-based near-infrared lightresponsive drug delivery systems for cancer treatment: A review - ScienceDirect. Journal of Materials Research Technology (2019) : 8: 1497 509.

23. R.Ali , H.Uzma , R.Tahir , B.Muhammad and H.Iqbal M N. Redox-responsive nano-carriers as tumor-targeted drug delivery systems. European Journal of Medicinal Chemistry (2018) : 157: S0223523418307025-.

24. Chunli, Shi, Xing, Guo, Qianqian, Qu, et al. Actively targeted delivery of anticancer drug to tumor cells by redox-responsive star-shaped micelles. Biomaterials ( 2014). 